\title{
Research Article \\ Effects of Head-Neck Positions on the Hand Grip Strength in Healthy Young Adults: A Cross-Sectional Study
}

\author{
Hamayun Zafar, Ahmad Alghadir (D), and Shahnawaz Anwer (i) \\ Rehabilitation Research Chair, College of Applied Medical Sciences, King Saud University, Riyadh, Saudi Arabia \\ Correspondence should be addressed to Shahnawaz Anwer; anwer_shahnawazphysio@rediffmail.com
}

Received 15 April 2018; Accepted 12 July 2018; Published 25 July 2018

Academic Editor: Imelda de Groot

Copyright (C) 2018 Hamayun Zafar et al. This is an open access article distributed under the Creative Commons Attribution License, which permits unrestricted use, distribution, and reproduction in any medium, provided the original work is properly cited.

\begin{abstract}
Background. Assessment of hand grip strength is vital for the evaluation of upper limb impairments and effective exercise prescription. Present study primarily aimed to investigate the effect of head-neck positions on the hand grip strength in healthy young adults. Secondarily, the present study compared hand grip strength between left versus right hand in different head-neck positions. Methods. Healthy young adults (age 19 - 30 year; $\mathrm{n}=40$ ) participated in this cross-sectional study. Hand grip strength was assessed in three head-neck positions (neutral, rotation left, and rotation right) using the standard adjustable Jamar hand dynamometer. Results. Hand grip strength in both sides (left and right) was greatest in the head-neck neutral position. Hand grip strength measured in head-neck left rotation position displayed the least strength in both sides. There was no significant difference noted between left and right side hand grip strength measured in head-neck neutral and right rotation positions. However, there was a significant difference noted between left and right side hand grip strength measured in head-neck left rotation positions. There was no significant effect of head-neck positions on hand grip strength noted in both sides. Conclusions. Hand grip strength was highest in the head-neck neutral position followed by head-neck rotation to the right. However, head-neck positions did not significantly affect hand grip strength in healthy young adults. Further studies assessing the hand grip strength in different neck positions in patients with neck pain and upper limb dysfunction may have significant implications for the assessment of hand grip strength.
\end{abstract}

\section{Background}

Assessment of hand grip strength is vital for the evaluation of upper limb impairments and effective exercise prescription [1, 2]. The hand is considered an important tool through which we manipulate and control our environment [3]. Previous studies recommended hand grip strength as a predictor of general health including heart disease [4], disability $[5,6]$, cognitive impairment $[5,6]$, cerebrovascular disease $[5,6]$, risk of future fracture $[7,8]$, and mortality $[9,10]$.

It was reported that body positions and upper limb positions can influence the measurement of hand grip strength $[11,12]$. The tonic neck reflex (TNR) is a reflex phenomenon in which head positions affect limb muscle tone [13]. Although it may influence all four limbs, its effect is more on the upper limbs than the lower limbs [3]. The TNR has two components including a symmetrical tonic neck reflex (STNR) and an asymmetrical tonic neck reflex (ATNR) [14]. Previous studies indicated that, even in healthy infants and adults, persistent
ATNR influences movements in specific states including motion stress and relaxation $[15,16]$.

Previously, Deutsch et al. [17] recommended the use of head-neck rotations during strengthening of upper extremity muscles to induce ATNR. Tokizane et al. [18] reported an influence of head-neck position on motor unit activity in infants, healthy adults, and neurologically impaired persons. In addition, Su et al. [19] reported an influence of shoulder and elbow positions on the hand grip strength in healthy adults. However, only few studies have investigated the influence of head-neck positions on the hand grip strength in healthy adults. Kumar et al. [3] reported that highest grip strength noted in head-neck rotation to left side. Similarly, Lee et al. [20] reported slightly increased grip strength measurement in head-neck rotation position compared to head-neck neutral in healthy adults. In contrast, Wong et al. [21] did not find an influence of head-neck rotation on the strength of the elbow flexors and extensors in healthy young women. 
The present study aimed to investigate the effect of head-neck positions on the hand grip strength in healthy young adults. Secondarily, present study compared hand grip strength between left versus right hand in different head-neck positions.

\section{Methods}

A total of 40 male and female students between the ages of 19 and 30 years participated in this cross-sectional study. Participants were excluded if they had any history of sensory deficits in upper extremity, history of recent upper extremity surgery, recent upper extremity injury, and undergoing any type resistance training. Study procedures were explained verbally to each participant after getting a written informed consent. The study was approved by the ethics committee of our institution.

Hand grip strength was assessed in three head-neck positions (neutral, rotation left, and rotation right) using the standard adjustable Jamar hand dynamometer as reported in the previous published studies [3, 17] (J-Tech 12-0259 Commander, Grip Track Dynamometer, Midvale, UT). The participants were asked to maintain the neck rotation at the maximal possible range. In addition to head-neck positions, the American Society of Hand Therapists' standardized grip-strength testing procedure was followed [22]. The dynamometer was fixed at the second handle position for measurement of hand grip strength. The participants were instructed to squeeze the handle of the dynamometer maximally and maintain it for 5 seconds. Average of the three measurements was recorded for the analysis purpose. A rest of one minute and five minutes was provided between three measurements and three different head-neck positions, respectively. Three head-neck positions were randomized to minimize fatigue effect.

All statistical tests were done using the SPSS (Version 22. IBM Inc., Chicago, IL). Descriptive statistics including proportion, mean, standard deviation, and 95\% confidence interval were presented. After testing normality of data, a repeated-measures analysis of variance (parametric tests) was used to compare hand grip strength in different head-neck positions. $t$-test was used to compare hand grip strength between left and right side in different head-neck positions. The results were considered statistically significant if $\mathrm{p}$ value was less than 0.05 .

\section{Results}

A total of 40 subjects between the ages of 19 and 30 years participated in this study. Table 1 gives the participants characteristics. Table 2 presents the comparison between left and right hand grip strength in different head-neck positions. Hand grip strength in both sides (left and right) was greatest in the head-neck neutral position. In addition, hand grip strength measured in head-neck left rotation position displayed least strength in both sides. There was no significant difference noted between left and right side hand grip strength measured in head-neck neutral and right rotation positions $(\mathrm{p}>0.05)$. However, there was a
TABLE 1: Participants characteristics.

\begin{tabular}{lc}
\hline Gender, No. (\%) & \\
Male & $26(65)$ \\
Female & $14(35)$ \\
\hline Age, years & $25.60(3.93)$ \\
Mean (SD) & $19-30$ \\
Range & \\
\hline Body mass index, Kg./m ${ }^{2}$ & $25.63(4.43)$ \\
Mean (SD) & $18.14-34.11$ \\
Range & $32(80)$ \\
Hand dominance, No. (\%) & $8(20)$ \\
Right & \\
Left &
\end{tabular}

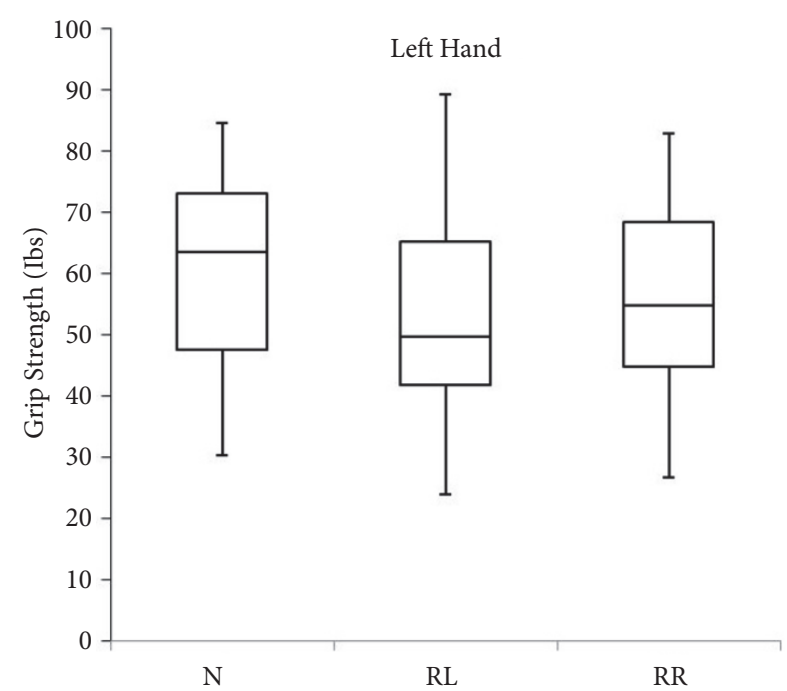

FIGURE 1: Box plot showing the distribution of hand grip strength in the left side measured in three neck positions (N, neutral; RL, rotation to left; $\mathrm{RR}$, rotation to right).

significant difference noted between left and right side hand grip strength measured in head-neck left rotation positions $(\mathrm{p}=0.023)$. Table 3 presents the comparison of hand grip strength in different head-neck positions using a repeatedmeasures analysis of variance. There was no significant effect of head-neck positions on hand grip strength noted in both sides $(p>0.05)$. Box plot showing the distribution of hand grip strength in the left and right side measured in three neck positions is presented in Figures 1 and 2.

\section{Discussion}

The primary aim of the present study was to investigate the effect of head-neck positions on the hand grip strength in healthy young adults. Secondarily, it compared hand grip strength between left versus right hand in different headneck positions. The results of present study indicated that the measurement of hand grip strength in the head-neck neutral position yields greatest strength in both sides (left and right). However, there was statistically insignificant effect 
TABLE 2: Comparison between left and right hand grip strength in different head-neck positions.

\begin{tabular}{lcccccc}
\hline & \multicolumn{3}{c}{ Hand grip strength (lbs), Left } & \multicolumn{3}{c}{ Hand grip strength (lbs), Right } \\
& Mean & SD & $95 \%$ CI & Mean & SD & 95\% CI \\
\hline N & 61.1 & 15.3 & $56.2-65.9$ & 62.6 & 17.4 & $57.3-67.8$ \\
RL & 53.5 & 15.6 & $48.7-58.4$ & 56.5 & 17.1 & $51.3-61.8$ \\
RR & 55.6 & 15.7 & $50.7-60.4$ & 57.8 & 15.9 & $52.6-63.1$ \\
\hline
\end{tabular}

*t-test (statistically significant if $\mathrm{p}<0.05)$; : neck in neutral position; RL: neck rotation left; RR: neck rotation right; CI: confidence interval.

TABLE 3: Comparison of hand grip strength in different head-neck positions using a repeated-measures analysis of variance.

\begin{tabular}{|c|c|c|c|c|c|}
\hline \multicolumn{6}{|c|}{ Tests of Inter-subjects Effects } \\
\hline \multicolumn{6}{|c|}{ Dependent Variable: Hand Grip Strength (lbs), Left side } \\
\hline Source & $\begin{array}{l}\text { Type III sum of } \\
\text { squares }\end{array}$ & df & Mean square & $\mathrm{F}$ & $* \mathrm{P}$ \\
\hline Corrected model & 1206.469 & 2 & 603.234 & 2.492 & .087 \\
\hline $\begin{array}{l}\text { Head-Neck Positions (N } \\
\text { vs RL vs RR) }\end{array}$ & 1206.469 & 2 & 603.234 & 2.492 & .087 \\
\hline Error & 28319.153 & 117 & 242.044 & & \\
\hline \multicolumn{6}{|c|}{ Dependent Variable: Hand Grip Strength (lbs), Right side } \\
\hline Corrected model & 813.802 & 2 & 406.901 & 1.434 & .242 \\
\hline $\begin{array}{l}\text { Head-Neck Positions (N } \\
\text { vs RL vs RR) }\end{array}$ & 813.802 & 2 & 406.901 & 1.434 & .242 \\
\hline Error & 33187.739 & 117 & 283.656 & & \\
\hline
\end{tabular}

$\mathrm{N}$ : neck in neutral position; RL: neck rotation left; RR: neck rotation right.

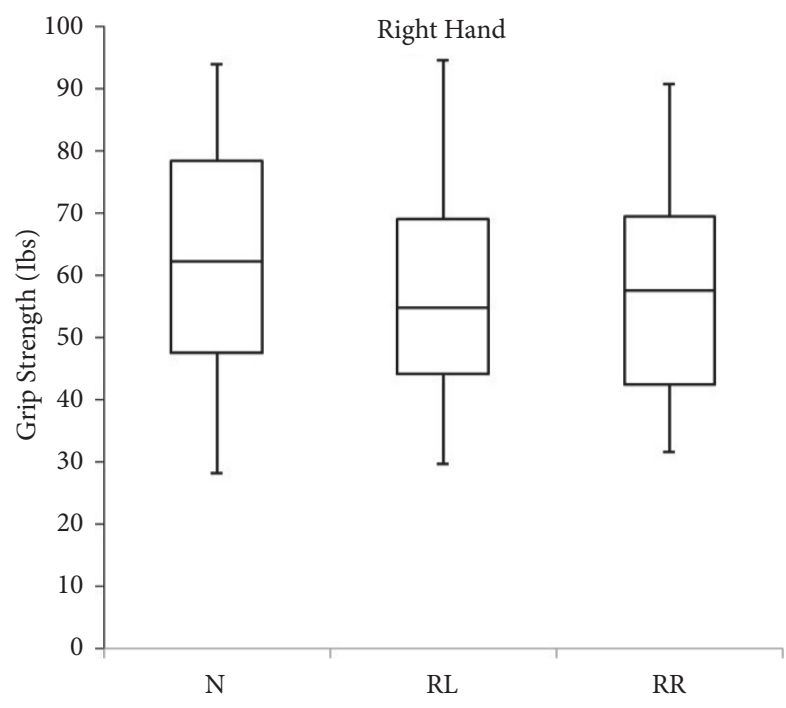

FIGURE 2: Box plot showing the distribution of hand grip strength in the right side measured in three neck positions (N, neutral; RL, rotation to left; $\mathrm{RR}$, rotation to right).

of head-neck positions on hand grip strength noted in both sides.

In contrast, Kumar et al. [3] reported highest grip strength measurement in head-neck rotation to left side. Similarly, Lee et al. [20] reported slightly better grip strength measurement in head-neck rotation position compared to headneck neutral in healthy adults. However, Wong et al. [21] did not find any effect of head-neck rotation on the strength of the elbow flexors and extensors in healthy young women. There were some methodological differences between Lee et al.'s study and the present study. In Lee et al.'s [20] study, the Kinesio tape was applied to the flexor muscle of the hand during the measurement of hand grip strength. This could be one of the reasons for different results. As per proprietor of the Kinesio tape, it improves muscle function by strengthening weak muscles $[23,24]$. In addition, Kinesio tape could cause physiological changes in the muscle and myofascial functions due to increase in the blood and lymph circulation at the taping area $[23,25]$. Moreover, it is also suggested that the application of Kinesio tape may stimulate cutaneous mechanoreceptors and, therefore, Kinesio tape might improve muscle excitability $[25,26]$. In the Kumar et al. [3] study, the participants were in the age group of 17-25 years; however, in the present study, the participant age was between 19 and 30 years. This might be one of the reasons for different results.

In this study, head-neck neutral and right rotation positions did not influence measurement of hand grip strength between left and right side. However, hand grip strength measured in head-neck left rotation position was significantly higher in the right side compared to the left side. This significant difference in grip strength (mean value of right is 56.5 compared to left $53.5 \mathrm{lbs}$ ) may be due to ATNR. Similarly the ATNR could have influenced greater mean value for left grip strength during neck rotation to right; but this possibly may not have occurred because the samples are comprised majorly $(80 \%)$ of right handed dominant volunteers. There could be another reason for such result; that is, the study 
conducted includes both genders grouped together with majorly $65 \%$ males and $35 \%$ females. Deutsch et al. [17] conclude that the influence of the tonic neck reflex may be elicited more easily in female volunteers than in males, which is primarily due to the reduced limb strength in females. A comparison between both the genders would have been more effective in reducing this biased result. In addition, hand grip strength was higher in head-neck right rotation position compared to left rotation in both hands. The mechanism of TNR is thought to come into play for this finding. Past studies showed that, even in healthy infants and adults, presence of ATNR could influences movements in particular states including movement and relaxation $[15,16]$.

This study is limited to only young adults. Therefore, similar study with older adults is warranted. In addition, further studies assessing the hand grip strength in different neck positions in patients with neck pain and upper limb dysfunction such as shoulder pain, elbow pain, and wrist pain may have significant implications for the assessment of hand grip strength.

\section{Conclusions}

The hand grip strength was highest in the head-neck neutral position followed by head-neck rotation to the right. However, head-neck positions did not significantly affect hand grip strength in healthy young adults.

\section{Abbreviations}

TNR: Tonic neck reflex

ATNR: Asymmetrical tonic neck reflex

STNR: Symmetrical tonic neck reflex.

\section{Data Availability}

The data used to support the findings of this study are available from the corresponding author upon request.

\section{Disclosure}

The funding body played no role in the study design, manuscript writing, or decision to submit the manuscript for publication.

\section{Conflicts of Interest}

The authors declare no conflicts of interest, financial or otherwise.

\section{Authors' Contributions}

Shahnawaz Anwer is corresponding author, participated in the study design, participated in the data collection, drafted the manuscript, and finalized the manuscript. Hamayun Zafar conceived the design, helped in data interpretation, and revised the manuscript critically. Ahmad Alghadir participated in the study design, helped with the ethics application, and revised the manuscript critically. All authors read and approved the final manuscript.

\section{Acknowledgments}

The authors are grateful to the Deanship of Scientific Research, King Saud University, for funding through Vice Deanship of Scientific Research Chairs.

\section{References}

[1] N. A. Incel, E. Ceceli, P. B. Durukan, H. Rana Erdem, and Z. Rezan Yorgancioglu, "Grip Strength: Effect of Hand Dominance," Singapore Medical Journal, vol. 43, no. 5, pp. 234-237, 2002.

[2] N. Massy-Westropp, W. Rankin, M. Ahern, J. Krishnan, and T. C. Hearn, "Measuring grip strength in normal adults: reference ranges and a comparison of electronic and hydraulic instruments," Journal of Hand Surgery, vol. 29, no. 3, pp. 514-519, 2004.

[3] . Kumar, "Grip Strength: Influence of Head-Neck Position in Normal Subjects," Journal of Neurology Research, 2012.

[4] K. Silventoinen, P. K. E. Magnusson, P. Tynelius, G. D. Batty, and F. Rasmussen, "Association of body size and muscle strength with incidence of coronary heart disease and cerebrovascular diseases: A population-based cohort study of one million Swedish men," International Journal of Epidemiology, vol. 38, no. 1, pp. 110-118, 2009.

[5] R. Cooper, D. Kuh, C. Cooper et al., "Objective measures of physical capability and subsequent health: a systematic review," Age and Ageing, vol. 40, no. 1, pp. 14-23, 2011.

[6] L. E. Hebert, P. A. Scherr, J. J. McCann, J. L. Bienias, and D. A. Evans, "Change in direct measures of physical performance among persons with Alzheimer's disease," Aging \& Mental Health, vol. 12, no. 6, pp. 729-734, 2008.

[7] P. M. Cawthon, R. L. Fullman, L. Marshall et al., "Physical performance and risk of hip fractures in older men," Journal of Bone and Mineral Research, vol. 23, no. 7, pp. 1037-1044, 2008.

[8] J. Sirola, T. Rikkonen, M. Tuppurainen, J. S. Jurvelin, E. Alhava, and H. Kröger, "Grip strength may facilitate fracture prediction in perimenopausal women with normal BMD: A 15year population-based study," Calcified Tissue International, vol. 83, no. 2, pp. 93-100, 2008.

[9] R. Cooper, D. Kuh, and R. Hardy, "Objectively measured physical capability levels and mortality: systematic review and meta-analysis," BMJ, vol. 341, article no. c4467, 2010.

[10] H. Sasaki, F. Kasagi, M. Yamada, and S. Fujita, "Grip Strength Predicts Cause-Specific Mortality in Middle-Aged and Elderly Persons," American Journal of Medicine, vol. 120, no. 4, pp. 337342, 2007.

[11] P. W. K. Fong and G. Y. F. Ng, "Effect of wrist positioning on the repeatability and strength of power grip," American Journal of Occupational Therapy, vol. 55, no. 2, pp. 212-216, 2001.

[12] V. Mathiowetz, K. Weber, G. Volland, and N. Kashman, "Reliability and validity of grip and pinch strength evaluations," Journal of Hand Surgery, vol. 9, no. 2, pp. 222-226, 1984.

[13] R. Magnus and A. de Kleijn, "The influence of the position of the head on the tone of the muscles of the extremities," in Pflugers Arch, vol. 145, pp. 548-455, 455, 1912.

[14] T. Fukuda, "Studies on human dynamic postures from the view point of postural reflexes," Acta Otolaryngol Suppl, vol. 161, pp. $1-52,1961$. 
[15] J. Coryell, A. Henderson, and J. Liederman, "Factors influencing the asymetrical tonic neck reflex in normal infants," Physical \& Occupational Therapy in Geriatrics, vol. 2, no. 2-3, pp. 51-65, 1982.

[16] R. Zemke, "Incidence of ATNR in normal preschool children," Physical \& Occupational Therapy In Pediatrics, vol. 1, pp. 31-37, 1980.

[17] H. Deutsch, H. Kilani, E. Moustafa, N. Hamilton, and J. P. Hebert Jr., "Effect of head-neck position on elbow flexor muscle torque production," Physical Therapy in Sport, vol. 67, no. 4, pp. 517-521, 1987.

[18] T. Tokizane, "Electromyographic studies on tonic neck, lumbar and labyrinthine reflexes in normal persons," The Japanese Journal of Physiology, vol. 2, pp. 130-146, 1951.

[19] M. Henkel, "Schrödinger invariance and strongly anisotropic critical systems," Journal of Statistical Physics, vol. 75, no. 5, pp. 1023-1061, 1994.

[20] J.-H. Lee, W.-G. Yoo, and K.-S. Lee, "Effects of head-neck rotation and Kinesio taping of the flexor muscles on dominanthand grip strength," Journal of Physical Therapy Science, vol. 22, no. 3, pp. 285-289, 2010.

[21] Y. C. R. Wong, D. Cameron, and R. W. Bohannon, "Elbow and hand muscle strength are not affected by head-neck position," Isokinetics and Exercise Science, vol. 7, no. 1, pp. 43-47, 1998.

[22] H. C. Roberts, H. J. Denison, H. J. Martin et al., "A review of the measurement of grip strength in clinical and epidemiological studies: towards a standardised approach," Age and Ageing, vol. 40, no. 4, pp. 423-429, 2011.

[23] K. Kase, H. Tatsuyuki, and O. Tomoki, Development of Kinesio ${ }^{\mathrm{TM}}$ Taping Perfect Manual, Kinesio ${ }^{\mathrm{TM}}$, Tokyo, 1996, 117-118.

[24] A. Yasukawa, P. Patel, and C. Sisung, "Pilot study: Investigating the effects of Kinesio Taping in an acute pediatric rehabilitation setting," American Journal of Occupational Therapy, vol. 60, no. 1, pp. 104-110, 2006.

[25] A. Yoshida and L. Kahanov, "The effect of kinesio taping on lower trunk range of motions," Research in Sports Medicine, vol. 15, no. 2, pp. 103-112, 2007.

[26] H. Murray and L. Husk, "Effect of kinesio taping on proprioception in the ankle," Journal of Orthopaedic \& Sports Physical Therapy, pp. 31-37, 2001. 


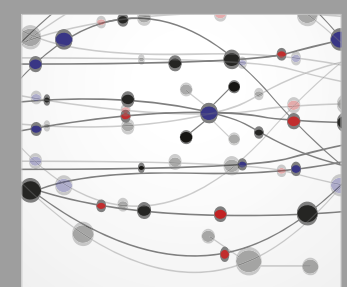

The Scientific World Journal
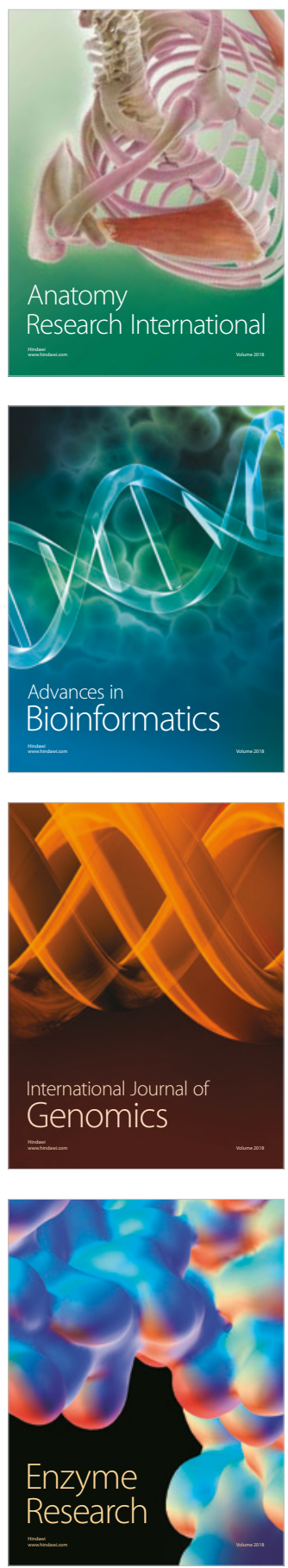
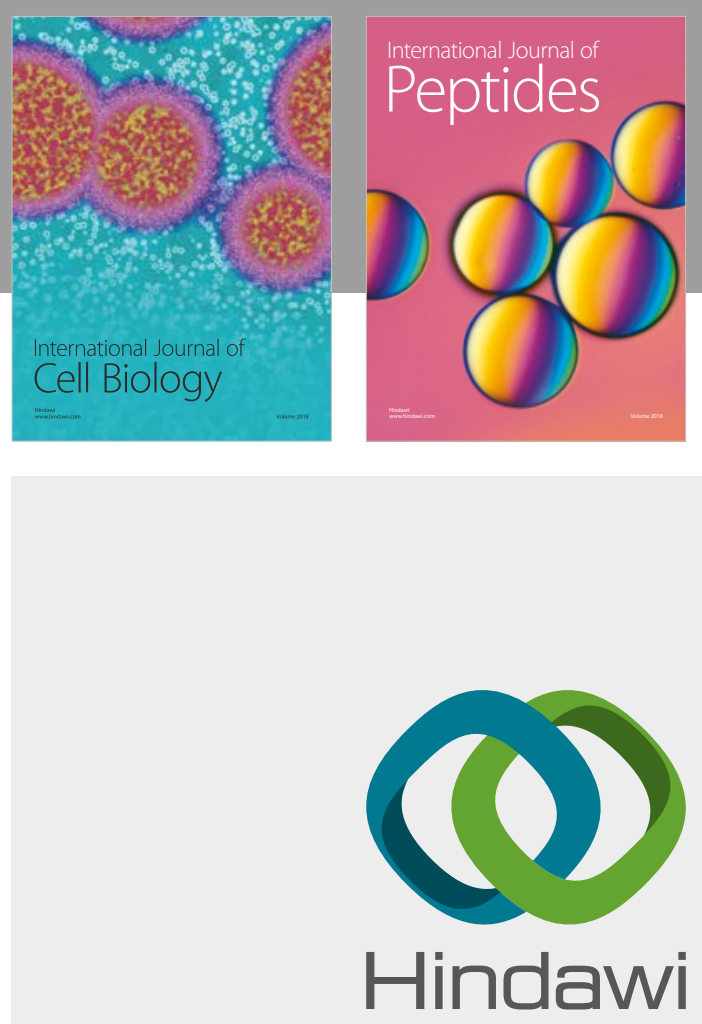

Submit your manuscripts at

www.hindawi.com
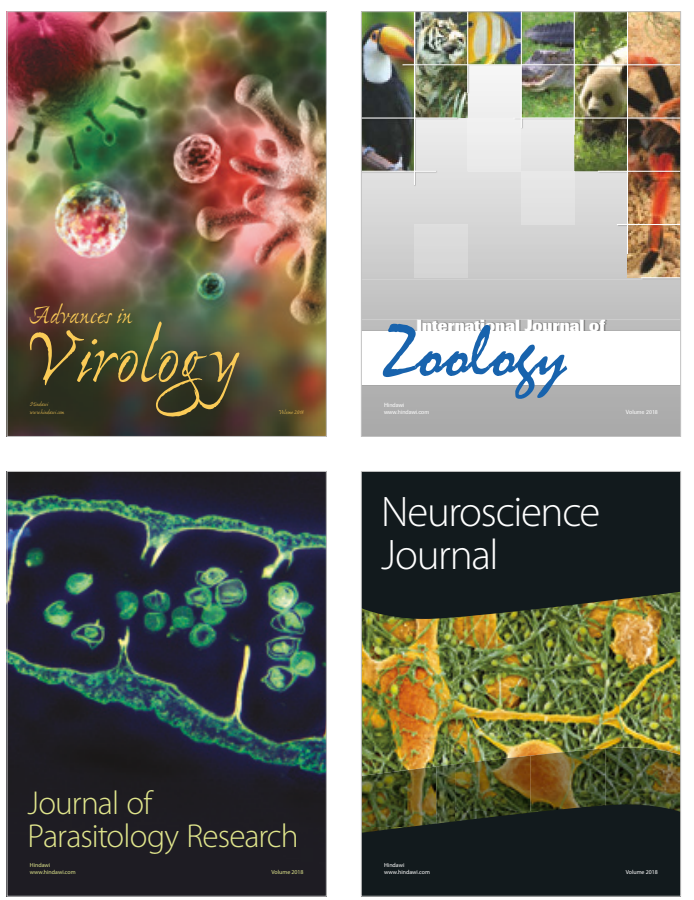
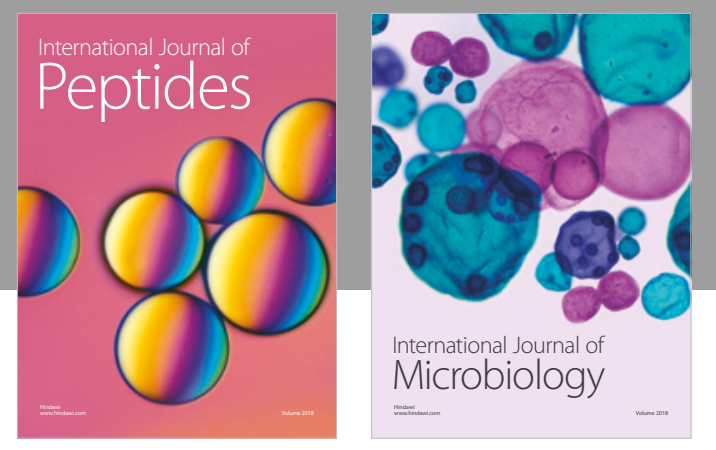

nternational Journal of Microbiology
Journal of
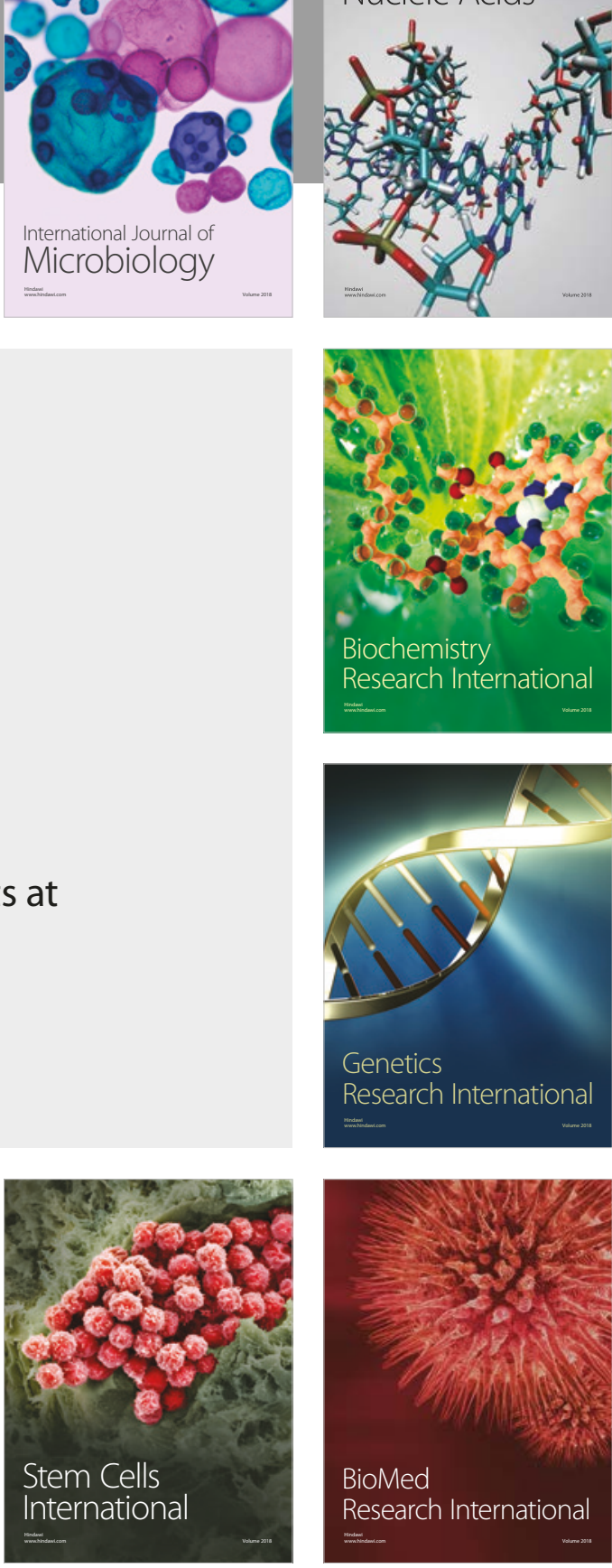
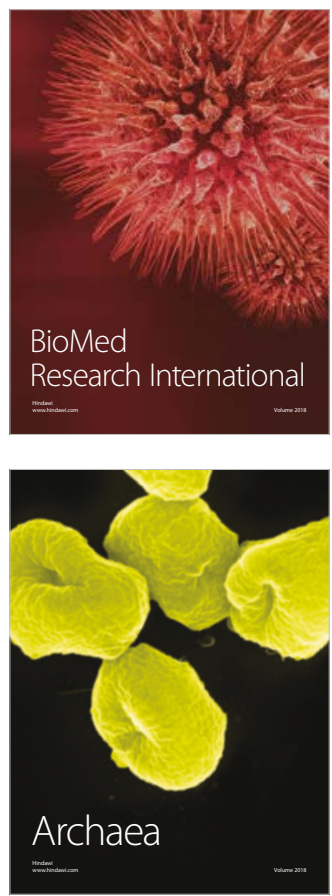